\title{
Effects of $\alpha$-phosphoglucomutase deficiency on cell wall properties and fitness in Streptococcus gordonii
}

Correspondence
Vladimir Lazarevic
vladimir.lazarevic@unil.ch

Received 27 June 2006

Revised 16 October 2006

Accepted 25 October 2006

\author{
Alain Bizzini, Paul Majcherczyk, Siham Beggah-Möller, Blazenka Soldo, \\ José M. Entenza, Muriel Gaillard, Philippe Moreillon and Vladimir Lazarevic
}

Département de Microbiologie Fondamentale, Bâtiment Biophore, Université de Lausanne, Quartier UNIL-Sorge, $\mathrm{CH}-1015$ Lausanne, Switzerland

\begin{abstract}
Streptococcus gordonii $\alpha$-phosphoglucomutase, which converts glucose 6 -phosphate to glucose 1 -phosphate, is encoded by pgm. The pgm transcript is monocistronic and is initiated from a $\sigma^{\mathrm{A}}$-like promoter. Mutants with a gene disruption in pgm exhibited an altered cell wall muropeptide pattern and a lower teichoic acid content, and had reduced fitness both in vitro and in vivo. In vitro, the reduced fitness included reduced growth, reduced viability in the stationary phase and increased autolytic activity. In vivo, the pgm-deficient strain had a lower virulence in a rat model of experimental endocarditis.
\end{abstract}

\section{INTRODUCTION}

$\alpha$-Phosphoglucomutase (PGM) converts glucose 6-phosphate to glucose 1-phosphate, which is the precursor of UDP-glucose. UDP-glucose serves as a glucosyl donor in several metabolic pathways. In Gram-positive bacteria, UDP-glucose may be used as the precursor of wall teichoic acids (WTAs), lipoteichoic acids, membrane glycolipids, capsules and exopolysaccharides (Boels et al., 2001; Lazarevic et al., 2005).

PGM deficiency has pleiotropic effects, the most common being morphological changes and slower growth (Hardy et al., 2000; Buchanan et al., 2005; Lazarevic et al., 2005). In the soil bacterium Bacillus subtilis, altered cell morphology of PGM-deficient mutants correlates with a deficiency in membrane glycolipids and is reversible by addition of $\mathrm{Mg}^{2+}$ (Lazarevic et al., 2005). Moreover, these mutants have a reduced ability to form biofilms and this may be attributed to their inability to synthesize a hypothetical glucosecontaining exopolysaccharide. Finally, changes in WTA content and structure, resulting from an inability to synthesize UDP-glucose, confer resistance to some phages.

In Streptococcus pneumoniae, an important cause of respiratory infections, PGM loss is associated with a reduced virulence, possibly due to a reduction in capsule production (Hardy et al., 2000, 2001). Similarly, in Streptococcus iniae, a fish pathogen that can cause invasive infection in humans,

Abbreviations: PGM, $\alpha$-phosphoglucomutase; PSD, post-source decay; WTA, wall teichoic acid.

The GenBank/EMBL/DDBJ accession number for the nucleotide sequence of Streptococcus gordonii pgm reported in this paper is D0.234767.
PGM deficiency correlates with diminished capsule production and reduced virulence, which might be due to an increased susceptibility to phagocytic clearance (Buchanan et al., 2005).

Streptococcus gordonii is a primary colonizer of the tooth surface, where it contributes to the formation of dental plaque. The bacterium may enter the bloodstream and, in susceptible individuals, cause infective endocarditis (Douglas et al., 1993). However, the use of S. gordonii strains harbouring foreign immunogenic peptides on their surface has been considered as an immunization technique (Pozzi et al., 1992). In the present work, we assessed the physiological implications of PGM in S. gordonii by studying the effects of PGM deficiency on in vitro and in vivo fitness, as well as on cell wall properties.

\section{METHODS}

Strains and growth conditions. S. gordonii Challis was routinely grown at $37^{\circ} \mathrm{C}$ without aeration, either in brain heart infusion (BHI) broth (Oxoid) or on BHI agar plates. Escherichia coli $\mathrm{DH} 5 \alpha$, used as a host for plasmid clones, was grown at $37^{\circ} \mathrm{C}$, either in Luria-Bertani (LB) broth (Difco) with aeration or on LB agar plates. Growth of the cultures was followed by monitoring $\mathrm{OD}_{600}$ with a Novaspec II spectrophotometer (Pharmacia LKB). When appropriate, erythromycin was added to the media at a final concentration of $5 \mu \mathrm{g} \mathrm{ml}^{-1}$ for S. gordonii. For E. coli transformants, ampicillin was used at a concentration of $50 \mu \mathrm{g} \mathrm{ml}{ }^{-1}$. Bacterial stocks were stored at $-80{ }^{\circ} \mathrm{C}$ in broth supplemented with $10 \%(\mathrm{v} / \mathrm{v})$ glycerol.

Construction of a pgm knockout mutant. The protein sequence deduced from the B. subtilis $p g c A$ gene encoding PGM was used to search for homology against the unfinished $S$. gordonii Challis genomic sequence available at The Institute for Genomic Research 
website (http://www.tigr.org). The 1977 bp region comprising the $S$. gordonii orthologue of $B$. subtilis pgcA was designated pgm, and it was PCR-amplified and sequenced; no discrepancies were found.

A 1038 bp pgm segment was PCR-amplified using oligonucleotides PGM_5' and PGM_3'_B, and cloned into pBAD-TOPO vector (Invitrogen) to obtain plasmid pBAD-TOPO_PGM. The $29 \mathrm{bp}$ internal part of the cloned pgm segment (residues 584-612 with respect to the putative pgm translational start) was excised by HindIII/ SalI and replaced by the 1083 bp erythromycin-resistance cassette, which includes its own promoter. The latter was obtained by the HindIII/SalI digestion of the PCR product generated with oligonucleotides VL602 and Ery_3'_SalI using pJDC9 DNA (Chen \& Morrison, 1988). The resulting plasmid pBAD-TOPO_ $\triangle$ PGM was digested with $M l u$ I and used to transform S. gordonii competent cells. Correct pgm knockout of $\triangle p g m:: e r m$, a randomly chosen erythromycin-resistant transformant, was verified by PCR (data not shown).

Transformation. S. gordonii competent cells were prepared as described by Pozzi et al. (1990). Transformants were selected on Columbia agar (Difco) plates supplemented with $3 \%$ human blood and $5 \mu \mathrm{g}$ erythromycin $\mathrm{ml}^{-1}$.

Oligonucleotides. The following oligonucleotides were used in this study:

Ery_3'_SalI, 5'-GTCGACCCTTGGAAGCTGTCAGTAGTATACCTA-3'; PGM_3'_B, 5' -AAAACGTTGAACATGGTTGC-3'; PGM_5', 5'-AAAGGAAGATGCCTTTTATACCAA-3'; VL602, 5' -AAAGCTTAAACGAAATGATACACCAATCAGTGCA-3'; VL837, 5' -TTGAGGTGGCGAACGGCGAATGAAAGTTCA-3'; VL838, 5' -TCGATAGCACGGATGTAAGTTGTCA-3'; VL840, 5'-CACTTCCACACCAACACGGTCAGCA-3'; VL851, 5' -GCCAAACCTTCAGTCGCTTGACGGACAACA-3'; VL852, 5'-CTGGAAGATCAGCGAAATCGA-3'; VL1008, 5'-GCATCAATATCTATGTTGTCCGTCA-3'. Underlined portions of the oligonucleotides represent engineered restriction sites: G/TCGAC, SalI; A/AGCTT, HindIII.

RNA extraction. Total RNA was extracted by using the RNeasy Protect Bacteria Mini Kit (Qiagen). Briefly, $4 \mathrm{ml}$ RNAprotect Bacteria Reagent (Qiagen) was added to a $2 \mathrm{ml}$ aliquot of S. gordonii culture in BHI broth and vortexed for $5 \mathrm{~s}$. After 5 min incubation at room temperature, the suspension was centrifuged at $3200 \mathrm{~g}$ for $10 \mathrm{~min}$. The supernatant was discarded, and the pellet was resuspended in $100 \mu \mathrm{l}$ TE buffer ( $10 \mathrm{mM}$ Tris, $1 \mathrm{mM}$ EDTA, pH 8.0) containing $15 \mathrm{mg}$ lysozyme $\mathrm{ml}^{-1}$ and incubated at room temperature for $15 \mathrm{~min}$. The manufacturer's protocol was followed from this point.

Mapping of transcriptional start site. The $5^{\prime}$ end of the pgm transcript was mapped with the BD SMART RACE cDNA Amplification Kit (BD Biosciences), according to the manufacturer's protocol. Briefly, gene-specific first-strand cDNA, synthesized from 560 ng total RNA extracted at an $\mathrm{OD}_{600}$ of 0.6 with pgm-specific primers VL838 and VL840, was tailed with the BD SMART II A oligonucleotide. The products were then amplified with a nested gene-specific primer VL837 and the Universal Primer A Mix in a PCR involving 30 cycles of denaturation at $95^{\circ} \mathrm{C}$ for $30 \mathrm{~s}$, annealing at $54^{\circ} \mathrm{C}$ for $30 \mathrm{~s}$ and extension at $72^{\circ} \mathrm{C}$ for $3 \mathrm{~min}$. The PCR product was purified with the GFX PCR DNA and Gel Band Purification Kit (Amersham Biosciences), and a nested PCR was carried out under the conditions described above using Nested Universal Primer A and oligonucleotide VL851. The nested PCR product was sequenced using primer VL852 (Synergene Biotech).

Northern blot. Total RNA was extracted from cells at an $\mathrm{OD}_{600}$ of 0.2. After incubation in $20 \mu \mathrm{l}$ (total volume) of denaturation buffer (1 M glyoxal, 25\% DMSO, $10 \mathrm{mM}$ sodium phosphate, $\mathrm{pH} 7.0)$ for $1 \mathrm{~h}$ at $50{ }^{\circ} \mathrm{C}$, total RNA $(10 \mu \mathrm{g})$ was separated by agarose electrophoresis and transferred onto a Hybond-N+ nylon membrane (Amersham Biosciences). RNA was blotted in $10 \times$ SSC with the VacuGene XL system (Amersham Biosciences) for $3.5 \mathrm{~h}$ under a vacuum of 50 mbar. After transfer, RNA was fixed to the membrane with a UV cross-linker (CX-2000, UVP) at a dose of $0.3 \mathrm{~J} \mathrm{~cm}^{-2}$. A 367 bp pgm probe labelled with digoxigenin was synthesized with a PCR DIG Labelling Mix (Roche Diagnostics) using oligonucleotide primers VL838 and VL1008. Hybridization and immunological detection were performed according to the supplier's instructions (Roche Diagnostics), using alkaline-phosphatase-conjugated antidigoxigenin Fab fragments to probe the digoxigenin-labelled DNA, and disodium 3-(4-methoxyspiro \{1,2-dioxetane-3,2'-(5' -chloro)tricyclo[3.3.1.1 $\left.1^{3,7}\right]$ decan $\left.\}-4-y l\right)$ phenyl phosphate (CSPD) as the reagent for chemiluminescent detection. Light emission was detected by using Hyperfilm ECL (Amersham Biosciences).

Cell extract preparation. Bacteria were grown in $200 \mathrm{ml} \mathrm{BHI}$ medium at $37^{\circ} \mathrm{C}$. At different points during growth, cells were recovered by centrifugation at $7000 \mathrm{~g}$ and $4{ }^{\circ} \mathrm{C}$. The pellets were rinsed with ice-cold distilled water and resuspended in $5 \mathrm{ml}$ $100 \mathrm{mM}$ triethanolamine, $\mathrm{pH} 7.4$, containing $5 \mathrm{mM} \mathrm{MgCl} 2$ and $2.2 \mathrm{mM}$ EDTA. The cells were disrupted by two passes through a One Shot cell disruptor (Constant Systems) at a pressure of $2.5 \mathrm{kbar}$ $(2.5 \mathrm{MPa})$. The cell debris was removed by centrifugation at $15000 \mathrm{~g}$ for $15 \mathrm{~min}$ and the supernatant was frozen at $-80^{\circ} \mathrm{C}$. The protein concentration was determined with the BCA Protein Assay Reagent (Pierce).

PGM assay. PGM activity was assayed as previously described (Lazarevic et al., 2005). The reaction mixture contained cell extract (20 $\mu$ g protein), $0.6 \mathrm{mM} \beta$-NADP, $25 \mu \mathrm{M}$ glucose 1,6-diphosphate and 1 unit glucose-6-phosphate dehydrogenase (Sigma), in $1 \mathrm{ml}$ (total volume) of $100 \mathrm{mM}$ triethanolamine, pH 7.4, containing $5 \mathrm{mM} \mathrm{MgCl} 2$ and $2.2 \mathrm{mM}$ EDTA. The reaction was started by adding $1.5 \mathrm{mM} \alpha$-D-glucose 1-phosphate. Under the assay conditions used, one unit of enzyme activity corresponds to the conversion of $1 \mathrm{nmol}$ substrate $\mathrm{min}^{-1}$ (mg cell protein $)^{-1}$.

Phase-contrast microscopy. Samples were fixed by addition of 0.1 vol. $20 \%$ formaldehyde and applied to a slide. Phase-contrast images were captured with a $\times 100$ Plan-Neofluar objective on a Axioskop 2 microscope (Carl Zeiss) equipped with MetaVue imaging software (version 6.1r5; Molecular Devices).

Viable cell estimation. The cell viability was estimated by using the LIVE/DEAD BacLight Bacterial Viability Kit for microscopy and quantitative assays (Molecular Probes). This kit differentially stains live or undamaged cells versus dead or damaged cells by using two nucleic acid stains. The Syto 9 green-fluorescent nucleic acid stain labels all cells whether living or dead, while the red-fluorescent nucleic acid stain propidium iodide enters only the cells with damaged membranes. The applicability of the kit to S. gordonii was verified with cells killed by incubation in $70 \%$ 2-propanol for $1 \mathrm{~h}$. Briefly, cells from samples of between 0.5 and $2 \mathrm{ml}$ were harvested by centrifugation for $10 \mathrm{~min}$ at $10000 \mathrm{~g}$, washed with $1 \mathrm{ml} 0.9 \%$ $\mathrm{NaCl}$ and resuspended in $200 \mu \mathrm{l} 0.9 \% \mathrm{NaCl}$. Syto 9 (component A) and propidium iodide (component B) were mixed at a $1: 1$ ratio and $0.6 \mu \mathrm{l}$ aliquots were added to each sample of cells. The suspensions were incubated for $15 \mathrm{~min}$ at room temperature in the dark. Bacteria were visualized with a DM IRE2 inverted laser scanning confocal microscope (Leica Microsystems) equipped with an HCX PL APO CS $\times 1001.4$ oil objective and Leica Confocal Software version 2.5. Images were taken using 488 and $543 \mathrm{~nm}$ laser excitations and 503-523 nm and 595-631 nm band-pass filters, for the Syto 9 and propidium iodide stains, respectively. The cells were counted in at least eight random fields and the proportion of viable or undamaged (green-stained) cells was calculated as a percentage of all the cells. 
Cell wall preparation. Cell walls were purified from bacteria grown to an $\mathrm{OD}_{600}$ of 0.3 in $\mathrm{BHI}$, as described by de Jonge et al. (1992).

Estimation of cell wall phosphate. Lyophilized cell walls were mineralized according to Ames (1966). A $50 \mu \mathrm{l}$ sample of a $1 \mathrm{mg} \mathrm{ml}^{-1}$ cell wall suspension was mixed with $50 \mu \mathrm{l} \quad 10 \%$ $\mathrm{Mg}\left(\mathrm{NO}_{3}\right)_{2}$ (w/v in $96 \%$ ethanol) in a borosilicate glass test tube and dried at $95^{\circ} \mathrm{C}$ for $3-4 \mathrm{~h}$. Samples were heated in a Bunsen flame for $2 \mathrm{~min}$. After cooling, $300 \mu \mathrm{l} 1 \mathrm{M} \mathrm{HCl}$ was added and tubes were heated for $15 \mathrm{~min}$ in a water bath at $100^{\circ} \mathrm{C}$. The phosphate concentration was determined according to Chen et al. (1956) against a standard curve generated using known concentrations of $\mathrm{K}_{2} \mathrm{HPO}_{4}$.

Muropeptide analysis. WTAs were removed from the cell walls by gently mixing the cell wall suspension in hydrofluoric acid (49\%) for $48 \mathrm{~h}$ at $4{ }^{\circ} \mathrm{C}$. The peptidoglycan was recovered, digested with mutanolysin (Sigma), and reduced and separated by HPLC, as described by de Jonge et al. (1992). The HPLC system (Hitachi) consisted of an L-7200 autosampler, an L-7100 gradient pump with low-pressure mixing and an L-7400 UV detector. Column temperature was maintained at $34^{\circ} \mathrm{C}$ using the $\mathrm{L}-7360$ column oven. Separation was performed by injection of a $100 \mathrm{ml}$ sample, containing $100 \mu \mathrm{g}$ of wall digest, onto a $250 \times 4.6 \mathrm{~mm}$ reverse-phase column (ODS-Hypersil $3 \mu \mathrm{m}$; Keystone Scientific). The material was eluted at $0.5 \mathrm{ml} \mathrm{min}^{-1}$ using a linear gradient of $5 \%(\mathrm{v} / \mathrm{v})$ methanol in $100 \mathrm{mM}$ sodium phosphate $(\mathrm{pH} 2.5)$ to $30 \%(\mathrm{v} / \mathrm{v})$ methanol in $100 \mathrm{mM}$ sodium phosphate $(\mathrm{pH} 2.8)$ over $160 \mathrm{~min}$. Eluted material was detected by its absorbance at $210 \mathrm{~nm}$. The results were analysed using the D-7000 HPLC System Manager program (Hitachi). Principal peaks were collected and desalted using C18 ZipTips (Millipore), according to the manufacturer's protocol. The mass of desalted peaks was determined by reflector positive-ion mode MALDI-TOF MS (Autoflex; Bruker Daltonics) using a 5-chloro-2mercaptobenzothiazole matrix in water, tetrahydrafuran and ethanol $(1: 1: 1)$. Pure peaks were sequenced by post-source decay (PSD) analysis (Xu et al., 1997).

Autolysis of whole cells. Cells were grown in BHI medium at $37^{\circ} \mathrm{C}$. At an $\mathrm{OD}_{600}$ of 0.2 , cells from a $3.5 \mathrm{ml}$ sample were recovered by centrifugation for $3 \mathrm{~min}$ at $10000 \mathrm{~g}$, washed with PBS buffer $\left(0.68 \% \mathrm{NaCl}, 0.148 \% \mathrm{Na}_{2} \mathrm{HPO}_{4}, 0.043 \% \mathrm{KH}_{2} \mathrm{PO}_{4}, \mathrm{pH} 7.2\right)$ and suspended in $1 \mathrm{ml} 30 \mathrm{mM}$ potassium phosphate ( $\mathrm{pH}$ 7.0). The suspension was incubated at $37^{\circ} \mathrm{C}$ and the $\mathrm{OD}_{600}$ was monitored. Results were expressed as percentages of the initial $\mathrm{OD}_{600}$.

Experimental endocarditis. Sterile aortic vegetations were produced in rats, as described by Heraief et al. (1982). Groups of animals were inoculated by intravenous challenge with $10^{6}$ c.f.u. exponential-phase streptococci. For the parent strain, this inoculum size produced endocarditis in $>90 \%$ of the rats, and thus it permitted a clear differentiation between virulent and putatively less virulent strains (Entenza et al., 1997; Stutzmann Meier et al., 2001). Rats were sacrificed $16 \mathrm{~h}$ after bacterial inoculation and viable colony counts in the vegetations and spleens were determined. The frequency of infection was compared by using Fisher's exact test. The median bacterial titres were compared by using a non-parametric Mann-Whitney unpaired test; $P<0.05$ was considered significant.

\section{RESULTS}

\section{Sequence analysis and expression of pgm}

The 572-residue protein encoded by the S. gordonii pgm gene showed $96.7 \%$ identity to the PGM of S. pneumoniae (Hardy et al., 2000) and $48.8 \%$ identity to the PGM of $B$. subtilis (Lazarevic et al., 2005). Analysis of the nucleotide sequence revealed that the $30 \mathrm{nt}$ segment located $8 \mathrm{nt}$ downstream of the S. gordonii pgm stop codon could form a stem-loop structure corresponding to a transcriptional terminator. The presence of a terminator-like sequence $193 \mathrm{nt}$ upstream of the pgm start codon, followed by a functional promoter (Fig. 1a; see below), suggests that pgm forms a one-gene operon. The Northern hybridization experiments yielded a major product of the expected length of about $1.8 \mathrm{~kb}$ (Fig. 1b), confirming a monocistronic transcription of $p g m$. The minor band of about $1.5 \mathrm{~kb}$ was most probably an artefact caused by the partially degraded mRNA and the abundance of 16S rRNA.

To identify promoters of the pgm gene, the $5^{\prime}$ end of the $p g m$ transcript was determined by $5^{\prime}$-RACE PCR, using RNA from exponentially growing $S$. gordonii cells. A band corresponding to a PCR product with an estimated length of $0.5 \mathrm{~kb}$ was revealed by agarose gel electrophoresis and sequenced (Fig. 2). The transcriptional start located $75 \mathrm{nt}$ upstream of the putative pgm start codon was preceded at a distance of $6 \mathrm{nt}$ by the sequence ATATGCTACAAT (Fig. 1a). This sequence exhibited a strong (10/11) adherence to the so-called extended -10 consensus sequence (RTRTGNTATAAT) of B. subtilis and S. pneumoniae $\sigma^{\mathrm{A}}$-type promoters (Helmann, 1995; Sabelnikov et al., 1995). Only a poorly matching $(3 / 6)-35$ region was found at the usual distance of $11 \mathrm{nt}$ upstream of the extended -10 . Therefore, it seems likely that, as in B. subtilis (Helmann, 1995) and S. pneumoniae (Sabelnikov et al., 1995), the extended -10 site may function without a recognizable -35 region. Moreover, in S. gordonii, transcription initiation from such promoters seems to be relatively frequent (M. Haenni and V. Lazarevic, unpublished).

\section{pgm knockout}

To confirm that pgm encodes PGM, we constructed a knockout mutant by inserting an erythromycin-resistance cassette into pgm and assayed cell extracts for PGM activity during the exponential phase of growth. In contrast to the parent strain (Fig. 3b), no detectable PGM activity was found in the $\Delta p g m:: \mathrm{erm}$ mutant, strongly suggesting that pgm encodes a unique PGM in S. gordonii. In the wild-type strain, after growth from an $\mathrm{OD}_{600}$ of 0.03 to a final reading of 1.39 , PGM activity increased by a factor of 2.3 .

\section{Cell morphology}

On solid BHI and blood agar, the pgm-deficient mutant formed somewhat smaller colonies than the parent strain (not shown). In liquid BHI medium, the pgm-deficient mutant grew at a slightly slower rate (mean doubling time of $59 \pm 3 \mathrm{~min}$, versus $48 \pm 1 \mathrm{~min}$ for the wild-type) and the $\mathrm{OD}_{600}$ in the stationary phase reached only $39 \%$ of the wildtype level (Fig. 3a).

Microscopic examination of the $\Delta p g m:$ : erm mutant grown in BHI liquid medium revealed an enlarged diameter 
(a)

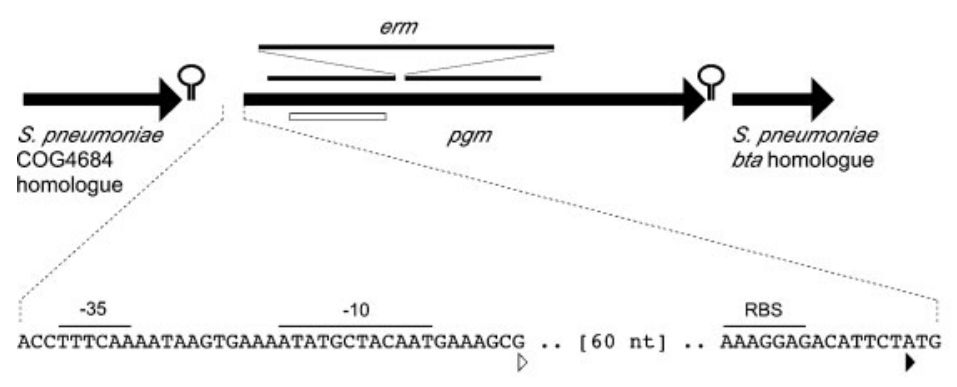

(b)

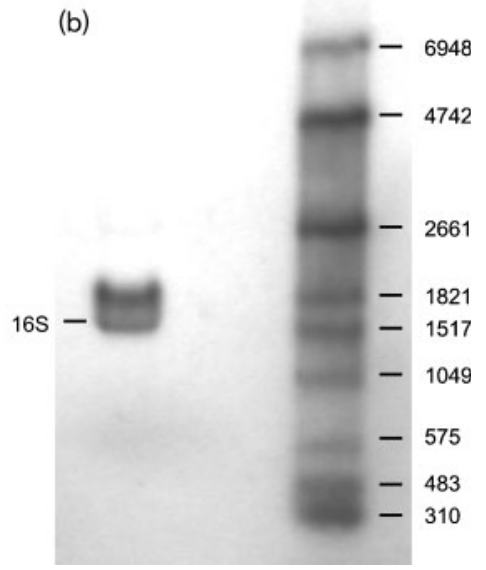

Fig. 1. Transcriptional unit of the pgm operon. (a) Gene organization of the chromosomal region containing pgm and the strategy for pgm inactivation. Arrows correspond to pgm and neighbouring genes. Stem-loop structures indicate possible transcriptional terminators. Insertion of the plasmid clone used for pgm inactivation is represented by bold horizontal lines. The -35 and extended -10 promoter regions and the ribosome-binding site (RBS) are labelled. Transcriptional and putative translational starts are indicated by white and black triangles, respectively. erm, erythromycin-resistance cassette. The DIGlabelled probe used for hybridization is indicated by a white bar. (b) Northern hybridization analysis of the pgm gene. The position of the 16S rRNA is shown on the left of the blot, and the sizes (nt) of the RNA molecular mass markers (Roche Diagnostics) are shown on the right.

compared with that of wild-type cells (Fig. 4). The cell size varied within the same chain. This aberrant cell morphology, which is in accordance with the observations recently made in S. iniae (Buchanan et al., 2005), was not reversible by addition of $\mathrm{Mg}^{2+}$, as shown for B. subtilis PGM-deficient mutants (Lazarevic et al., 2005).

\section{Cell viability in vitro}

The viability of the $\Delta p g m:: \mathrm{erm}$ mutant was investigated using the LIVE/DEAD BacLight Bacterial Viability Kit, which includes stains to determine whether cells have an intact or damaged membrane. In the exponential phase, at an $\mathrm{OD}_{600}$ of $0.3,99$ and $93 \%$ of the wild-type and $\mathrm{pgm}$ deficient cells, respectively, were stained as viable (Table 1). In contrast, a striking difference in viability was observed after $24 \mathrm{~h}$ batch cultivation. Wild-type cells showed only a $2 \%$ decline in the viable count, whereas the pgm-deficient mutant exhibited $>30 \%$ loss of viability under the same conditions.

\section{In vivo virulence}

The role of PGM was further evaluated in a rat model of experimental endocarditis. The results are presented in Table 2. Whereas the parent strain infected $100 \%$ of both vegetations and spleens, the $p g m$ deletion mutant infected only $54 \%$ of these tissues $(P<0.05)$. Moreover, the rats inoculated with the parent strain had significantly higher vegetation and spleen bacterial densities than those inoculated with the $\Delta p g m:$ :erm mutant $(P<0.05)$. Thus, in agreement with previous findings demonstrating that mutations in PGM had a significant effect on the virulence of S. pneumoniae and S. iniae in different animal models (Buchanan et al., 2005; Hardy et al., 2001), our results showed that the S. gordonii $\Delta p g m$ : : erm mutant was also less virulent in experimental endocarditis.

\section{Cell autolysis and cell wall analyses}

The autolysis of whole cells was monitored in terms of the decrease in $\mathrm{OD}_{600}$ of exponentially grown bacteria (a)

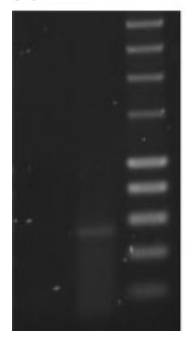

(b)

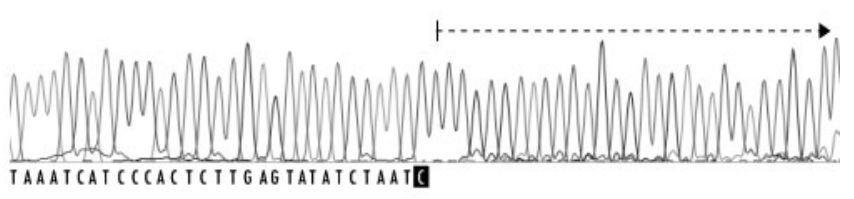

Fig. 2. Determination of the transcriptional start site by 5'-RACE PCR. (a) Agarose gel electrophoresis of PCR-amplified cDNA tailed with BD SMART II Oligonucleotide. DNA was stained with SYBR Safe and visualized under UV light. (b) A chromatogram from the sequencing of the 5'-RACEPCR product. The nucleotide complementary to the start of the transcript is highlighted and it is followed by the BD SMART II Oligonucleotide tail (dashed arrow). 


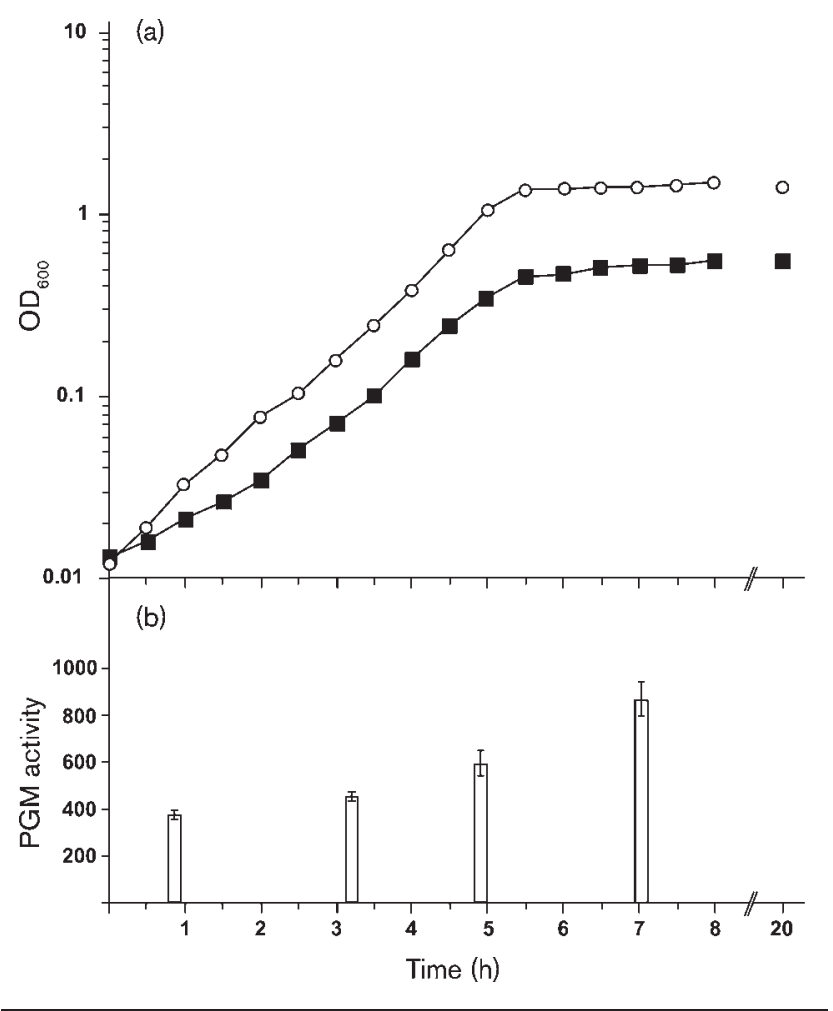

Fig. 3. Growth in $\mathrm{BHI}$ medium and change in $\mathrm{PGM}$ activity during growth. (a) Overnight cultures grown in $\mathrm{BHI}$ at $37^{\circ} \mathrm{C}$ were diluted into fresh medium to an $\mathrm{OD}_{600}$ of $0.01-0.013$. Cultures were incubated at $37^{\circ} \mathrm{C}$ and the $\mathrm{OD}_{600}$ of the wildtype ( $\bigcirc$ ) and pgm-deficient strains ( $\square$ ) was monitored. (b) PGM activity [nmol $\mathrm{min}^{-1}$ ( $\mathrm{mg}$ cell protein) ${ }^{-1}$ ] and error bars (SD) are indicated for the wild-type strain only, since activity was not detected in the $\Delta p g m:: e r m$ strain. Samples were taken from exponential $\left(O D_{600} 0.03,0.2\right.$ and 1.0) and stationary growth phases $\left(O D_{600} 1.39\right)$.

resuspended in a hypotonic phosphate buffer. In these conditions, the $p g m$-deficient strain lysed at a faster rate than the wild-type (Fig. 5). The increased autolysis might be due to an altered cell wall structure that allows hydrolytic enzymes increased access to the peptidoglycan.
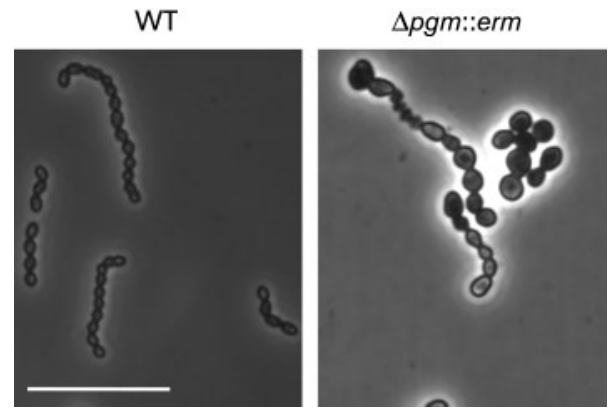

Fig. 4. Cell morphology of the wild-type (WT) and mutant $S$. gordonii strains. Cells were grown overnight in $\mathrm{BHI}$ medium at $37^{\circ} \mathrm{C}$. Bar, $10 \mu \mathrm{m}$.

In order to check whether the mutant had an altered cell wall structure, its cell wall muropeptide pattern was compared with that of its parent. Cell wall muropeptides were prepared from both the mutant and the parent on three different occasions and analysed separately. Both the parent and the mutant had complex muropeptide patterns that did not vary between culture days (Fig. 6). The composition of the components present in individual peaks was determined (Table 3). Identical structures were present in both the parent and the mutant, but the relative amounts varied between strains (Fig. 6, Table 4). These differences were quantified by calculating the relative amounts of the different peaks labelled in Fig. 6. Peaks 1, 2, 5b and 7 were significantly increased in the $\Delta p g m:: \mathrm{erm}$ strain compared with the wild-type, whereas peaks 3, 4, 5a and 6 were significantly decreased. No significant difference was observed in the amounts of peak $5 \mathrm{c}$ found in either strain (Table 4). Peaks 1, 2, 3, 4, 6 and 7 were found to be pure by MALDI-TOF MS and were identified by PSD analysis (Table 3). Peaks 5a, 5b and 5c contained several components and were not further analysed in this study. The fact that peak 2 was more abundant in the mutant than in the parent, and that it was a simple monomer, suggests that the wall of the mutant might be simpler than that of the parent.

To test whether the pgm knockout affects the synthesis of a cell wall anionic polymer, we compared the cell wall

Table 1. Effect of the pgm knockout on viability and cell wall phosphate content

\begin{tabular}{|lccc|}
\hline Strain & \multicolumn{2}{c}{ Viable cells $(\%)^{*}$} & $\begin{array}{c}\text { Cell wall phosphate }[\mu \mathrm{mol} \\
\left.(\mathbf{m g} \text { cell wall })^{-1}\right] \dagger\end{array}$ \\
\cline { 2 - 3 } & Exponential phase & Stationary phase & \\
\hline Wild-type & $99.1 \pm 0.3$ & $96.8 \pm 1.8$ & $0.221 \pm 0.018$ \\
$\Delta p g m:: \mathrm{erm}$ & $92.5 \pm 0.1$ & $60.8 \pm 1.8$ & $0.153 \pm 0.013$ \\
\hline
\end{tabular}

${ }^{*}$ Mean $( \pm \mathrm{SD})$ of three independent experiments.

$\dagger$ Mean $( \pm \mathrm{SD})$ based on three independent measurements obtained on two cell wall preparations. 
Table 2. Infectivity rate and bacterial densities in vegetation and splenic tissues of rats $16 \mathrm{~h}$ after bacterial challenge with an inoculum of $10^{6}$ c.f.u.

\begin{tabular}{|lccccc|}
\hline \multirow{2}{*}{ Strain } & \multicolumn{2}{c}{ Vegetation tissue } & & \multicolumn{2}{c|}{ Splenic tissue } \\
\cline { 2 - 3 } \cline { 6 - 7 } & $\begin{array}{c}\text { No. infected } \\
\text { rats/total }(\%)\end{array}$ & $\begin{array}{c}\text { Median }(\text { range }) \\
{\left[\log _{10}\left(\text { c.f.u. } \mathbf{g}^{-1}\right)\right]}\end{array}$ & & $\begin{array}{c}\text { No. infected } \\
\text { rats/total }(\%)\end{array}$ & $\begin{array}{c}\text { Median }(\text { range }) \\
{\left[\log _{10}\left(\text { c.f.u. }^{-1}\right)\right]}\end{array}$ \\
\hline Wild-type & $10 / 10(100 \%)$ & $6.67(4.05-7.63)$ & & $10 / 10(100 \%)$ & $3.12(2.08-3.72)$ \\
$\Delta p g m:: \mathrm{erm}$ & $6 / 11(54 \%)^{*}$ & $4.11(2.00-7.44)^{*}$ & & $6 / 11(54 \%)^{*}$ & $1.66(1.00-2.60)^{*}$ \\
\hline
\end{tabular}

${ }^{\star} P<0.05$ versus wild-type $S$. gordonii.

phosphate content of the $\Delta p g m:: \mathrm{erm}$ and the wild-type strains. The phosphate content of the wild-type (Table 1) was very close to $0.23 \mu \mathrm{mol}$ phosphate $\left(\mathrm{mg}\right.$ cell wall) ${ }^{-1}$, a value that has been reported for S. gordonii NCTC 7865 (Rose et al., 1997). Phosphate measurements revealed about $30 \%$ reduction in the cell wall of the PGM-deficient mutant (Table 1). This decreased value is to be expected for walls impoverished in sugar phosphate polymers.

\section{DISCUSSION}

S. gordonii deficient in PGM, the enzyme providing the link between glycolysis and biosynthetic processes requiring UDP-glucose, exhibited multiple phenotypic changes. These changes, especially the reduced survival of the organisms in stationary phase, increased autolytic activity and reduced virulence in infective endocarditis, point to a global loss of bacterial fitness.

Other studies have shown that at least part of the virulence attenuation observed in PGM-deficient $S$. iniae and $S$.

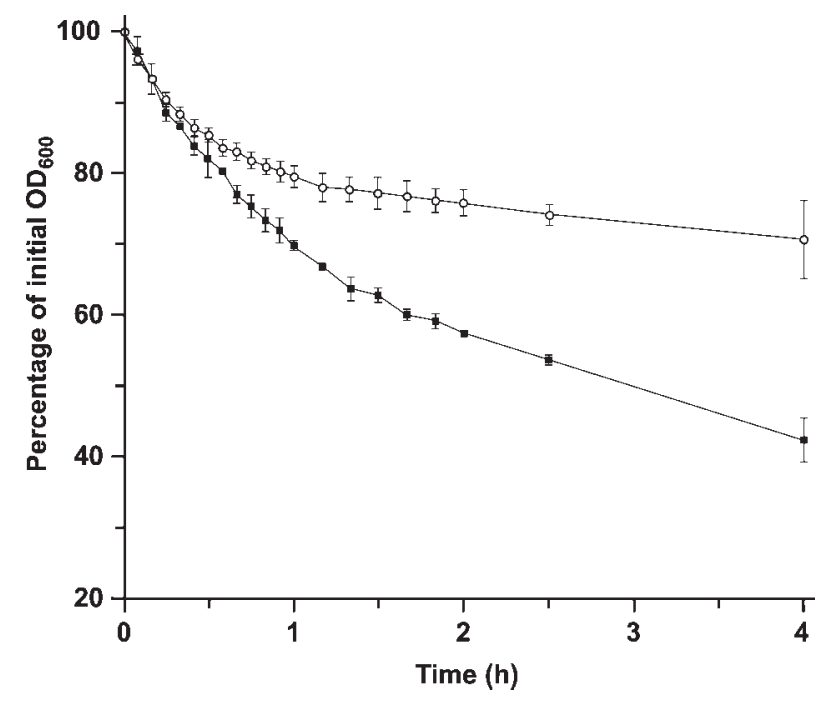

Fig. 5. Autolysis at $\mathrm{pH} 7.0$ of whole cells from an exponentialphase culture of $S$. gordonii. The mean and SEM were calculated from values obtained in three experiments. $\bigcirc$, Wild-type; 口, $\Delta$ pgm::erm mutant. pneumoniae is likely to be due to a diminished capsule expression (Buchanan et al., 2005; Hardy et al., 2001). Interestingly, S. gordonii appears to be an unencapsulated bacterium. Therefore, the reduced in vivo and in vitro fitness in the PGM-deficient mutant may be caused by changes in other cell envelope components.

The cell wall of most Gram-positive bacteria is composed of peptidoglycan, which is a structurally defining cell wall polymer that contains covalently attached anionic polymers, such as WTA and teichuronic acid. The repeating units of most WTAs contain substituted polyol phosphate. WTAs contribute to the cell wall electrolyte properties, modify the activity of peptidoglycan hydrolases and maintain cation homeostasis (Neuhaus \& Baddiley, 2003). In the rod-shaped

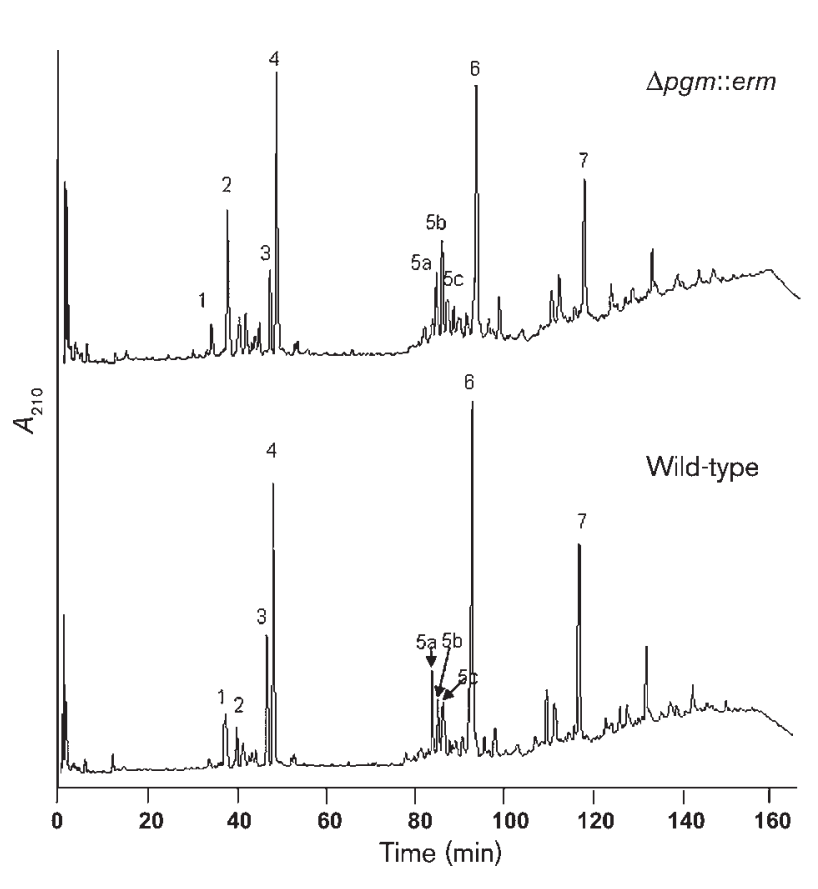

Fig. 6. Muropeptide fingerprints of the wild-type S. gordonii and its PGM-deficient mutant. Principal peaks numbered were analysed by MALDI-TOF MS. The mutant ( $\Delta$ pgm::erm) and parent (WT) contained the same peaks, but the proportion of certain peaks varied between the strains, notably the increase of peak 2 in the PGM-deficient mutant. 
Table 3. Molecular mass and composition of pure muropeptides from wild-type and $\Delta$ pgm : : erm peptidoglycan

Principal peaks were analysed by MALDI-TOF MS. Peaks 1, 2, 3, 4, 6 and 7 were pure and were identified by PSD analysis. Peaks $5 \mathrm{a}, 5 \mathrm{~b}$ and $5 \mathrm{c}$ contained several components and were not further analysed in this study. Identical structures were present in the parent and mutant. $[\mathrm{M}+\mathrm{Na}]^{+}$, mass of sodium adduct; G-M, $N$-acetylglucosamine- $N$-acetylmuramic acid; Ala, alanine; Glx, glutamine/glutamate; Lys, lysine; ND, not determined.

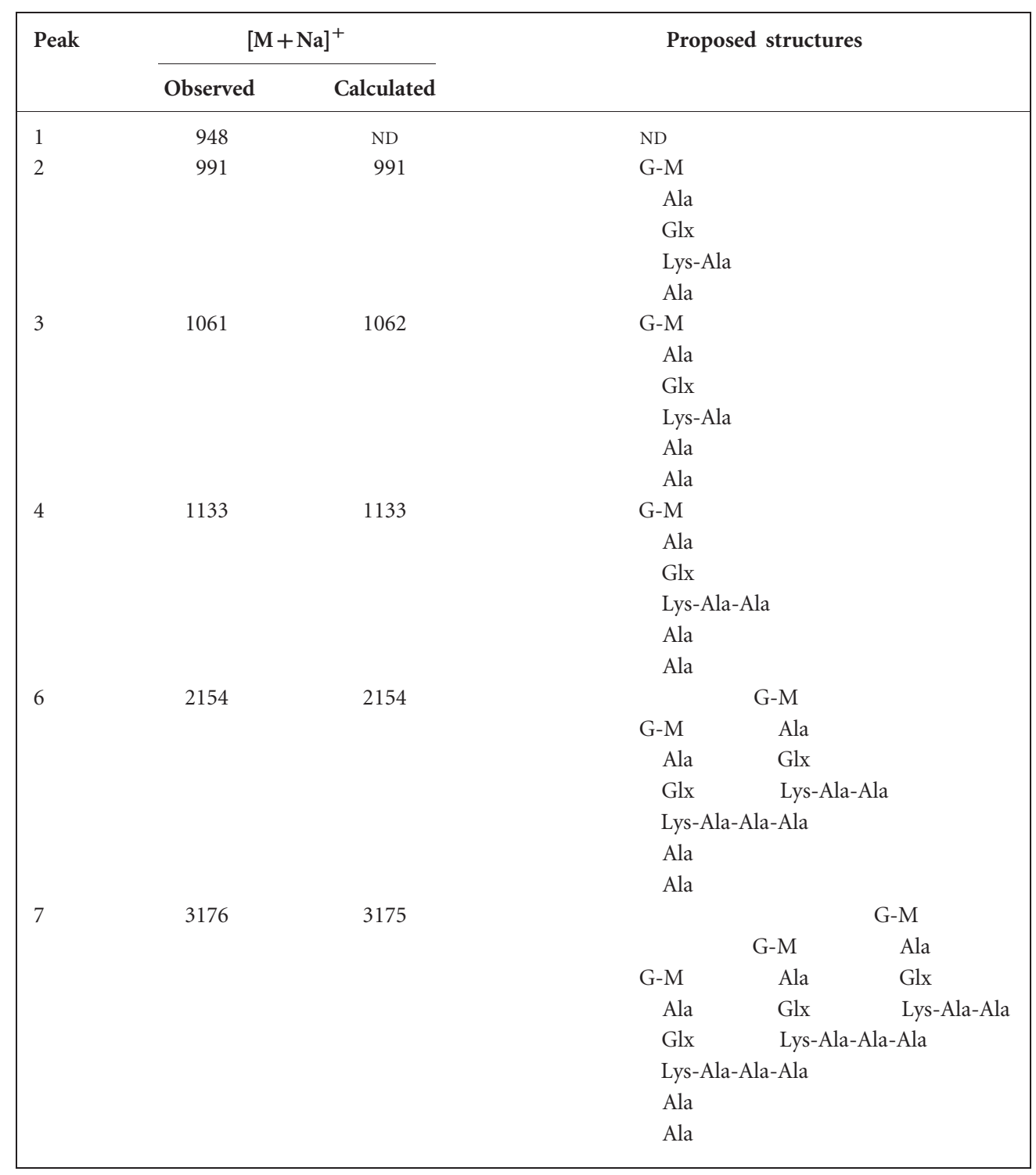

bacterium B. subtilis, as well as in the spherical Streptococcus oralis, WTA deficiency correlates with abnormal shape and cell growth (Horne \& Tomasz, 1993; Soldo et al., 2002). Since most species of viridans streptococci do not contain polyol-WTA (Rose et al., 1997), their sugar-phosphate polymers probably perform some of the roles carried out by WTA. Moreover, Hancock \& Baddiley (1985) proposed that these so-called sugar 1-phosphate polymers should be recognized as WTAs. Although the structure of S. gordonii Challis sugar-phosphate polymer has not been determined, it may be similar to those found in the viridans group of streptococcal strains whose repeated unit is a glucose- and phosphate-containing hexasaccharide or heptasaccharide (Cisar et al., 1997). Therefore, the PGM deficiency that prevents the synthesis of UDP-glucose, which is an important glucosyl donor for the synthesis of a variety of cell wall polymers, may lead to the abnormality in sugarphosphate polymer metabolism and, as a consequence, altered cell morphology.

Since PGM does not appear to be involved in the muropeptide biosynthetic pathway, PGM deficiency does 
Table 4. Muropeptide composition of the parent and PGMdeficient strains

Muropeptides were prepared from the wild-type and $\Delta p g m:$ erm strains on three separate occasions. Each batch of muropeptides was analysed in duplicate by HPLC. The values are percentages of the sum of all integrated peak areas presented in Table 3 and they are means \pm SEM of the six analyses. The percentage peak areas were compared by Student's two-tailed distribution test.

\begin{tabular}{|lrrcc|}
\hline Peak & Wild-type & $\boldsymbol{\Delta} \boldsymbol{p g m}: \mathbf{:} \boldsymbol{e r m}$ & Variation factor $^{*}$ & Pvalue \\
\hline 1 & $1.63 \pm 0.7$ & $2.95 \pm 0.47$ & 1.81 & 0.001 \\
2 & $3.90 \pm 1.4$ & $11.21 \pm 0.96$ & 2.87 & 0.000 \\
3 & $8.54 \pm 0.2$ & $5.14 \pm 0.36$ & 0.60 & 0.000 \\
4 & $17.83 \pm 0.8$ & $15.67 \pm 0.65$ & 0.88 & 0.002 \\
$5 \mathrm{a}$ & $7.65 \pm 0.5$ & $7.04 \pm 1.09$ & 0.92 & 0.000 \\
$5 \mathrm{~b}$ & $5.64 \pm 0.6$ & $8.11 \pm 0.20$ & 1.44 & 0.001 \\
$5 \mathrm{c}$ & $7.59 \pm 0.6$ & $7.34 \pm 0.67$ & 0.97 & 0.396 \\
6 & $28.93 \pm 2.1$ & $21.25 \pm 2.71$ & 0.73 & 0.003 \\
7 & $18.29 \pm 1.5$ & $21.29 \pm 3.04$ & 1.16 & 0.052 \\
\hline
\end{tabular}

${ }^{*}$ Factor by which the composition of the muropeptide of $\Delta p g m:$ :erm differs from the wild-type.

not provide a straightforward explanation for changes in the muropeptide pattern of the $\Delta p g m:$ erm strain. Moreover, we are not aware of any other study linking PGM deficiency to a change in the muropeptide pattern in other bacterial species. One possibility is that changes in WTA metabolism may influence the activity of peptidoglycan-hydrolytic or -biosynthetic enzymes, resulting in an altered muropeptide composition and an increased autolysis rate.

It has been hypothesized that the altered cell morphology in a PGM-deficient $B$. subtilis strain might be due to deficiencies in glucose 1-phosphate metabolites, monoglucosyldiacylglycerol and/or diglucosyldiacylglycerol (Lazarevic et al., 2005). In B. subtilis, synthesis of these glycolipids requires YpfP, a processive enzyme that catalyses successive addition of glucosyl residues from UDP-glucose to diacylglycerol (Jorasch et al., 1998). Interestingly, sequence similarity searches failed to reveal orthologues of $B$. subtilis $y p f P$ in $S$. gordonii and other streptococci. Therefore, the synthesis of membrane glycolipids in streptococci seems to involve a family of enzymes that are evolutionarily distinct from those operating in bacilli and staphylococci. Nevertheless, a deficiency in membrane glycolipids may be due to an inability to synthesize the putative glycosyl donor UDP-glucose.

S. gordonii is a commensal oral bacterium that has been considered as a potential live vaccine vector in humans (Pozzi et al., 1992; Kotloff et al., 2005). It may be theoretically advantageous to use a pgm-deficient $S$. gordonii as a vector in cases where only transient antigen exposure is required. An attenuated strain would be naturally outcompeted by the resident microflora, preventing long-term antigen stimulation. The use of such a strain may also reduce the risk of infective endocarditis after accidental entry of the bacterium into the bloodstream. Whether or not the diminished virulence properties conferred by pgm deficiency may offer an advantage for the use of S. gordonii as a potential vaccine vector in humans warrants further investigation.

\section{ACKNOWLEDGEMENTS}

This work was supported by grants $3235-62^{\prime} 698$ (MD/PhD programme, to A. B.), 3200B0-103793 (to P. M.) and 3100A0-102205 (to V. L.), from the Swiss National Science Foundation. We thank Marisa Haenni for communicating unpublished results, and Marlyse Giddey, Stéphanie Rosset, Jacques Vouillamoz and Jean-Luc Barblan for outstanding technical assistance.

\section{REFERENCES}

Ames, B. N. (1966). Assay of inorganic phosphate, total phosphate and phosphatases. Methods Enzymol 8, 115-118.

Boels, I. C., Ramos, A., Kleerebezem, M. \& de Vos, W. M. (2001). Functional analysis of the Lactococcus lactis galU and galE genes and their impact on sugar nucleotide and exopolysaccharide biosynthesis. Appl Environ Microbiol 67, 3033-3040.

Buchanan, J. T., Stannard, J. A., Lauth, X., Ostland, V. E., Powell, H. C., Westerman, M. E. \& Nizet, V. (2005). Streptococcus iniae phosphoglucomutase is a virulence factor and a target for vaccine development. Infect Immun 73, 6935-6944.

Chen, J. D. \& Morrison, D. A. (1988). Construction and properties of a new insertion vector, pJDC 9 , that is protected by transcriptional terminators and useful for cloning of DNA from Streptococcus pneumoniae. Gene 64, 155-164.

Chen, P. S., Toribara, T. Y. \& Warner, H. (1956). Microdetermination of phosphorus. Anal Chem 18, 1756-1758.

Cisar, J. O., Sandberg, A. L., Reddy, G. P., Abeygunawardana, C. \& Bush, C. A. (1997). Structural and antigenic types of cell wall polysaccharides from viridans group streptococci with receptors for oral actinomyces and streptococcal lectins. Infect Immun 65, 5035-5041.

de Jonge, B. L., Chang, Y. S., Gage, D. \& Tomasz, A. (1992). Peptidoglycan composition of a highly methicillin-resistant Staphylococcus aureus strain. The role of penicillin binding protein 2A. J Biol Chem 267, 11248-11254.

Douglas, C. W., Heath, J., Hampton, K. K. \& Preston, F. E. (1993). Identity of viridans streptococci isolated from cases of infective endocarditis. J Med Microbiol 39, 179-182.

Entenza, J. M., Caldelari, I., Glauser, M. P., Francioli, P. \& Moreillon, P. (1997). Importance of genotypic and phenotypic tolerance in the treatment of experimental endocarditis due to Streptococcus gordonii. J Infect Dis 175, 70-76.

Hancock, I. C. \& Baddiley, J. (1985). Biosynthesis of the bacterial envelope polymers teichoic acid and teichuronic acid. In The Enzymes of Biological Membranes, pp. 279-307. Edited by A. N. Martonosi. New York: Plenum.

Hardy, G. G., Caimano, M. J. \& Yother, J. (2000). Capsule biosynthesis and basic metabolism in Streptococcus pneumoniae are linked through the cellular phosphoglucomutase. J Bacteriol 182, 1854-1863.

Hardy, G. G., Magee, A. D., Ventura, C. L., Caimano, M. J. \& Yother, J. (2001). Essential role for cellular phosphoglucomutase in virulence of type 3 Streptococcus pneumoniae. Infect Immun 69, 2309-2317. 
Helmann, J. D. (1995). Compilation and analysis of Bacillus subtilis $\sigma^{\mathrm{A}}$-dependent promoter sequences: evidence for extended contact between RNA polymerase and upstream promoter DNA. Nucleic Acids Res 23, 2351-2360.

Heraief, E., Glauser, M. P. \& Freedman, L. R. (1982). Natural history of aortic valve endocarditis in rats. Infect Immun 37, 127-131.

Horne, D. S. \& Tomasz, A. (1993). Possible role of a cholinecontaining teichoic acid in the maintenance of normal cell shape and physiology in Streptococcus oralis. J Bacteriol 175, 1717-1722.

Jorasch, P., Wolter, F. P., Zahringer, U. \& Heinz, E. (1998). A UDP glucosyltransferase from Bacillus subtilis successively transfers up to four glucose residues to 1,2-diacylglycerol: expression of $y p f P$ in Escherichia coli and structural analysis of its reaction products. Mol Microbiol 29, 419-430.

Kotloff, K. L., Wasserman, S. S., Jones, K. F., Livio, S., Hruby, D. E., Franke, C. A. \& Fischetti, V. A. (2005). Clinical and microbiological responses of volunteers to combined intranasal and oral inoculation with a Streptococcus gordonii carrier strain intended for future use as a group A streptococcus vaccine. Infect Immun 73, 2360-2366.

Lazarevic, V., Soldo, B., Medico, N., Pooley, H., Bron, S. \& Karamata, D. (2005). Bacillus subtilis $\alpha$-phosphoglucomutase is required for normal cell morphology and biofilm formation. Appl Environ Microbiol 71, 39-45.

Neuhaus, F. C. \& Baddiley, J. (2003). A continuum of anionic charge: structures and functions of D-alanyl-teichoic acids in Grampositive bacteria. Microbiol Mol Biol Rev 67, 686-723.
Pozzi, G., Musmanno, R. A., Lievens, P. M., Oggioni, M. R., Plevani, P. \& Manganelli, R. (1990). Method and parameters for genetic transformation of Streptococcus sanguis Challis. Res Microbiol 141, 659-670.

Pozzi, G., Contorni, M., Oggioni, M. R., Manganelli, R., Tommasino, M., Cavalieri, F. \& Fischetti, V. A. (1992). Delivery and expression of a heterologous antigen on the surface of streptococci. Infect Immun $\mathbf{6 0}$, 1902-1907.

Rose, R. K., Matthews, S. P. \& Hall, R. C. (1997). Investigation of calcium-binding sites on the surfaces of selected Gram-positive oral organisms. Arch Oral Biol 42, 595-599.

Sabelnikov, A. G., Greenberg, B. \& Lacks, S. A. (1995). An extended -10 promoter alone directs transcription of the DpnII operon of Streptococcus pneumoniae. J Mol Biol 250, 144-155.

Soldo, B., Lazarevic, V., Pooley, H. M. \& Karamata, D. (2002). Characterization of a Bacillus subtilis thermosensitive teichoic aciddeficient mutant: gene mnaA $(y v y H)$ encodes the UDP- $N$-acetylglucosamine 2-epimerase. J Bacteriol 184, 4316-4320.

Stutzmann Meier, P., Entenza, J. M., Vaudaux, P., Francioli, P., Glauser, M. P. \& Moreillon, P. (2001). Study of Staphylococcus aureus pathogenic genes by transfer and expression in the less virulent organism Streptococcus gordonii. Infect Immun 69, 657-664.

Xu, N., Huang, Z. H., de Jonge, B. L. \& Gage, D. A. (1997). Structural characterization of peptidoglycan muropeptides by matrix-assisted laser desorption ionization mass spectrometry and postsource decay analysis. Anal Biochem 248, 7-14.

Edited by: J. C. Paton 